\title{
Fruit flies in space
}

The US National Aeronautics and Space Administration (NASA) plans to launch its new Fruit Fly Lab (FFL) to the International Space Station (ISS) in 2014 as part of a mission to assess the effects of microgravity and other aspects of prolonged space travel on the organisms. NASA hopes the mission will help scientists to understand how longterm space flight might affect humans.

Because fruit flies resemble humans at the genetic, cellular and biological systems levels, they make good models for biomedical research. But their small size and short generation time make them particularly good models for biomedical research in space. This is partly because space is hard to come by in space, with many projects competing for berths in the ISS. Thousands of fruit flies can be housed in a small cassette, similar in size to a deck of cards. This allows scientists to design experiments involving thousands of individuals, enough to give the tests a high degree of statistical power to detect relevant



effects. Furthermore, the effects of space flight can be monitored throughout the flies' entire lifespan and even over multiple generations during a single mission.

Fruit flies were the first animals to be launched into space. In 1947, they blasted off in a $\mathrm{V}-2$ rocket, reaching an altitude of about 68 miles in less than 200 seconds before returning to Earth by parachute.

In contrast, the FFL was custom-designed by scientists at NASA's Ames Research Center (Moffett Field, CA) led by Sharmila Bhattacharya. For the new mission (called
FFL-01), it will be transported to the ISS aboard the SpaceX Dragon capsule while carrying about 200 fruit flies; about 10,000 insects are expected to come back 30 days later at the close of the mission. The FFL features NanoRacks centrifuges that allow for flies to be exposed to variable gravity conditions from $1 \mathrm{~g}$ (Earth gravity) to fractional $g$ (moon or Mars gravity) to $0 \mathrm{~g}$ (weightlessness or microgravity). This enables the use of an in-space control group of flies, whereas previous experiments used a control group housed on Earth instead.

FFL-01 will follow up on results from a 2006 experiment aboard the Discovery Space Shuttle showing that fruit flies reared in space had compromised immune systems (PLoS ONE 6, 1-10; 2011). A second mission, planned for 2015, will assess space travel's effects on the fruit flies' cardiovascular systems. NASA has invited researchers to submit proposals for future projects using the FFL.

\section{Monica Harrington}

\section{CHILLY MICE CONFOUND CANCER STUDIES}

International and US guidelines for the housing of laboratory mice dictate that temperatures be kept constant and in the range of $20-26{ }^{\circ} \mathrm{C}$. When given a choice, mice instead prefer temperatures of $\sim 30^{\circ} \mathrm{C}$; this is the temperature at which they can maintain homeostasis without expending extra energy to combat cold stress. So why are lab mice housed at lower temperatures? One reason is that laboratory staff are more comfortable at room temperature, given the heavy protective clothing they are required to wear. Other reasons for lower temperatures are also practical, such as improved breeding efficiency and reduced need for frequent cage cleaning.

But Elizabeth Repasky of the Roswell Park Cancer Institute in Buffalo, NY, and others have suggested that housing mice at these low temperatures does more than just cause the mice to experience cold stress: it influences research results as well. Testing four different mouse cancer models, scientists in Repasky's lab found that mice had slower tumor formation and reduced metastasis when housed at $30-31{ }^{\circ} \mathrm{C}$ than when housed at $22-23{ }^{\circ} \mathrm{C}$ (Proc. Natl. Acad. Sci. USA published online 18 November 2013; doi:10.1073/pnas.1304291110).

Furthermore, their immune responses were also more robust. The warmer mice showed higher numbers of CD8+ lymphocytes, activated CD8+ T cells and white blood cells when compared with the chillier mice. The warmer mice also had lower counts of myeloid-derived suppressor cells that often suppress immune responses to cancer. The authors therefore attributed the difference in tumor growth to the increased antitumor immune response seen in the warmer mice. This idea is further supported by their finding that mice with compromised immune systems showed no temperature-dependent difference in tumor growth. "I don't think any one of us predicted how many fundamental aspects of the antitumor immune response would be affected by simply feeling cold," Repasky told Nature News.

The findings have important implications, particularly for studies of cancer drugs that target the immune system. Furthermore, Repasky says her research sheds light on "why people who seem to be a lot more chronically stressed than others do more poorly in response to not only their cancer risk but also their overall outcome after therapy." But the study's findings also indicate how the way in which research animals are housed can have an important effect on research outcomes. "I think we're really undermining the ability of these models to be accurate," Repasky told The Scientist. 